\title{
Coupling Constants for Scalar Glueball Decay*
}

\author{
J. Sexton ${ }^{\dagger}$, A. Vaccarino ${ }^{\ddagger}$ and D. Weingarten \\ IBM Research, P.O. Box 218, Yorktown Heights, NY 10598
}

\begin{abstract}
We evaluate the partial decay widths of the lightest scalar glueball to pairs of pseudoscalar quark-antiquark states. The calculation is done in the valence (quenched) approximation on a $16^{3}$ lattice at $\beta=5.7$. These predictions and values obtained earlier for the infinite volume continuum limit of the scalar glueball's mass are in good agreement with the observed properties of $f_{J}(1710)$ and inconsistent with all other observed meson resonances.
\end{abstract}

\section{Introduction}

Whether or not glueballs have been observed in experiment is still generally considered unsettled. Since the properties of glueballs are not expected to be drastically different from the properties of flavor singlet bosons including valence quarks and antiquarks, the identification in experiment of states with large glueball contributions is difficult without a reliable calculation of the predictions of QCD. The lattice formulation of QCD appears to us to give the most reliable method now available for determining QCD's predictions for the masses and decay couplings of glueballs.

For the infinite volume continuum limit of the valence (quenched) approximation to the lightest scalar glueball mass, we reported some time ago [1] the value $1740(71) \mathrm{MeV}$. This prediction was obtained using ensembles of 25000 to 30000 gauge configurations on each of several different lattices. An earlier independent valence approximation calculation [2] of the scalar glueball mass, extrapolated to the continuum limit [3] following Ref. [1], yields a prediction of $1625(94) \mathrm{MeV}$. The calculation of Ref [2] uses ensembles of between 1000 and 3000 configurations on several different lattices. Combining the two mass calculations and taking into account the correlation between their statistical uncertainties arising from a com-

\footnotetext{
*Talk presented by D. Weingarten

${ }^{\dagger}$ Permanent address: Department of Mathematics, Trinity College, Dublin 2, Republic of Ireland

${ }^{\ddagger}$ Present address: Piazza Giovanetti 1, Novara, 28100 Italy
}

mon procedure for converting lattice quantities into physical units gives a scalar glueball mass of $1707(64) \mathrm{MeV}$. This result and the mass prediction with larger statistical weight are both in good agreement with the mass of $f_{J}(1710)$ and are strongly inconsistent with all but $f_{0}(1500)$ [4] among the other established flavor singlet resonances which could be scalars. For $f_{0}(1500)$ the disagreement is still by more than three standard deviations.

The valence approximation, in effect, replaces the momentum and frequency dependent color dielectric constant arising from quark-antiquark vacuum polarization with its zero-momentum, zero-frequency limit [5]. For the long distance properties of hadrons, we would expect this approximation to be fairly reliable. The infinite volume continuum limits, for example, of the valence approximation to the masses of eight low lying hadrons composed of quarks and antiquarks differ from experiment by amounts ranging up to $6 \%$ [6]. A $6 \%$ error in the glueball mass would be $100 \mathrm{MeV}$. An adaptation of an argument giving a negative sign for the valence approximation error in $f_{\pi}$ [6] also suggests a negative sign for the glueball mass error. Thus we would expect the scalar glueball in full QCD to lie between $1707(64) \mathrm{MeV}$ and $1807(64) \mathrm{MeV}$, again favoring $f_{J}(1710)$ with $f_{0}(1500)$ still possible but improbable.

The key question not answered by the mass results, however, is whether the lightest scalar glueball has a decay width small enough for this particle actually to be identified in experiment. It seems likely to us that a scalar glueball with a width of a few hundred $\mathrm{MeV}$ or less and mass 
in the neighborhood of $1700 \mathrm{MeV}$ would already have been seen in experiment. Alternatively, if the scalar glueball has a width of a $\mathrm{GeV}$ or more, the prospect of ever finding this particle seems remote. A further question in the identification of $f_{J}(1710)$ as a glueball is raised by the argument that since glueballs are flavor singlets they should have the same couplings to $2 \pi_{0}$, to $2 K_{L}$, and to $2 \eta$. This equality is violated by $f_{J}(1710)$ decay couplings.

In the present article we report the first lattice QCD calculation of the valence (quenched) approximation to the decay couplings of the lightest scalar glueball to pairs of pseudoscalar quarkantiquark states. The couplings constants we obtain, combined with the mass prediction of $1740(71) \mathrm{MeV}$, give a total two-pseudoscalar decay width of $108(29) \mathrm{MeV}$. With any reasonable guess concerning the scalar glueball's branching fraction to multibody decay modes, the resulting total decay width is well below $200 \mathrm{MeV}$ and therefore small enough for the scalar glueball to be identified in experiment. In fact, the predicted total two-pseudoscalar decay width, and individual couplings to $2 \pi_{0}$, to $2 K_{L}$, and to $2 \eta$ are all in good agreement with properties of $f_{J}(1710)$ and inconsistent with all other established flavor singlet resonances which could be scalars. The total two-body width of $f_{J}(1710)$ is $99(15) \mathrm{MeV}[8]$. A comparison of our predicted coupling constants with data for $f_{J}(1710)$ [8] is shown in Figure 1.

The calculation we present uses 10500 independent gauge configurations on a lattice $16^{3} \times 24$ at $\beta=5.70$. The corresponding inverse lattice spacing is $1.35 \mathrm{GeV}$. We believe this lattice has spacing sufficiently small and volume sufficiently large to give partial widths within $30 \%$ of their infinite volume continuum limits.

In the valence approximation, according to one point of view, glueballs are pure field and are not mixed with states including valence quarks or antiquarks. The agreement between the glueball mass and decay couplings found in the valence approximation and the observed mass and decay couplings of $f_{J}(1710)$ appears to us to be strong evidence that this state is largely a scalar glueball with at most some relatively smaller amplitude for configurations including valence quark-

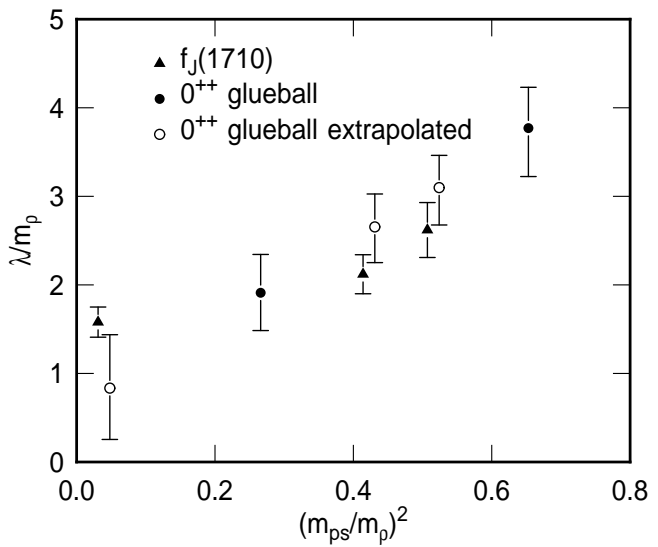

Figure 1. Couplings for decay to a pair of pseudoscalars as a function of pseudoscalar mass squared.

antiquark pairs.

The calculations presented here were carried out on the GF11 parallel computer [9] at IBM Research and took approximately two years to complete at a sustained computation rate of between 6 and 7 Gflops. A preliminary version of this work is discussed in Ref. [10].

In the remainder of this paper we describe our method for determining scalar glueball decay couplings then present our numerical results.

\section{Method}

We work with a euclidean lattice gauge theory on a lattice $L^{3} \times T$, with the plaquette action for the gauge field and the Wilson action for quarks. We assume initially exact flavor SU(3) symmetry for the quark mass matrix. Each gauge configuration is fixed to Coulomb gauge. We then define a collection of smeared fields. We describe smearing only for the particular choice of parameters actually used in the decay calculation. Let $U_{i}(x)$ for a space direction $i=1,2,3$, be a smeared link field [i] given by the average of the 9 links in direction $i$ from the sites of the ( 3 site) $\mathrm{x}(3$ site) square oriented in the two positive space directions orthogonal to $i$ starting at site $x$. Let $V_{i j}(x)$ be the trace of the product of $U_{i}(x)$ and $U_{j}(x)$ around the outside of a (3 link) $\mathrm{x}$ (3 link) square. Let $g(t)$, carrying zero momentum, be the sum of the $V_{i j}(x)$ for all $i, j$ and $x$ with time component $t$. Define $\bar{\Psi}(x)$ and $\Psi(x)$ to be quark and antiquark 
fields, respectively, smeared [6] by convoluting the local fields with a space direction gaussian with mean-square radius 6.0. The smeared field $\pi_{i}(x)$ with flavor index $i$ is $\bar{\Psi}(x) \gamma^{5} \Lambda_{i} \Psi(x)$, where $\Lambda_{i}$ is a Gell-Mann flavor matrix. Define $\tilde{\pi}_{i}(\vec{k}, t)$ to be the Fourier transform of $\pi_{i}(x)$ on the time $t$ lattice hyperplane.

$E_{1}^{\pi}$ and $E_{2}^{\pi}$ are defined as the energy of a single pseudoscalar at rest or with momentum magnitude $|\vec{k}|=2 \pi / L$, respectively. The field strength renormalization constant $\eta_{1}^{\pi}$ is defined by setting the large $t$ asymptote of the vacuum expectation value $<\tilde{\pi}_{i}^{\dagger}(0, t) \tilde{\pi}_{i}(0,0)>$ to $\left(\eta_{1}^{\pi}\right)^{2} L^{3} \exp \left(-E_{1}^{\pi} t\right)$. Define $\eta_{2}^{\pi}$ similarly from a pseudoscalar field with momentum magnitude $|\vec{k}|=2 \pi / L$. The glueball mass $E^{g}$ and field strength renormalization constant $\eta^{g}$ are defined by setting the large $t$ asymptote of $<g(t) g(0))>$ to be $\left(\eta^{g}\right)^{2} L^{3} \exp \left(-E^{g} t\right)$.

Let the flavor singlet, rotationally invariant, two-pseudoscalar field $\Pi\left(t_{1}, t_{2}\right)$ be $(16)^{-1 / 2} \sum_{i} \pi_{i}\left(0, t_{1}\right) \pi_{i}\left(0, t_{2}\right)$, with the sum over $i$ running from 1 to 8 . Define the flavor singlet field for two zero momentum pseudoscalars $\tilde{\Pi}_{1}\left(t_{1}, t_{2}\right)$ as $(16)^{-1 / 2} \sum_{i} \tilde{\pi}_{i}\left(0, t_{1}\right) \tilde{\pi}_{i}\left(0, t_{2}\right)$. Let the field $\tilde{\Pi}_{2}\left(t_{1}, t_{2}\right)$ for two pseudoscalars with opposite momenta be $(24)^{-1 / 2} \sum_{i \vec{k}} \tilde{\pi}_{i}\left(\vec{k}, t_{1}\right) \tilde{\pi}_{i}\left(-\vec{k}, t_{2}\right)$ where the sum for $\vec{k}$ is over the three positive orientations with $|\vec{k}|=2 \pi / L$.

Define $\mid 1>$ and $\mid 2>$, respectively, to be the lowest and second lowest energy flavor singlet, rotationally invariant two-pseudoscalar states, both normalized to $1 . \quad E_{i}^{\pi \pi}$ is the energy of $|i\rangle$. The amplitude $\eta_{i j}^{\pi \pi}(t)$ is then defined as $L^{-3}<$ $i\left|\tilde{\Pi}_{j}(t, 0)\right| \Omega>$. At large $t, \eta_{i j}^{\pi \pi}(t)$ approachs $\eta_{i j}^{\pi \pi} \exp \left(-E_{j}^{\pi} t\right)$. We expect the diagonal coefficients $\eta_{11}^{\pi \pi}$ and $\eta_{22}^{\pi \pi}$ to be larger than the offdiagonal $\eta_{21}^{\pi \pi}$ and $\eta_{12}^{\pi \pi}$, respectively. Since pairs of pseudoscalars can interact and scatter, however, the off-diagonal coefficients need not be zero.

The connected three-point functions $T_{i}\left(t_{g}, t_{\pi}\right)$ from which we extract coupling constants are $<g\left(t_{g}\right) \tilde{\Pi}_{i}\left(t_{\pi}, 0\right)>-<g\left(t_{g}\right)><\tilde{\Pi}_{i}\left(t_{\pi}, 0\right)>$. If the hopping constant $\kappa$ is chosen so that $E_{1}^{\pi \pi}=$ $E^{g}$, the lightest intermediate state which can appear between the glueball and pseudoscalars in $T_{1}\left(t_{g}, t_{\pi}\right)$ is $\mid 1>$. For large $t_{g}$ with $t_{\pi}$ fixed, $T_{1}\left(t_{g}, t_{\pi}\right)$ will therefore be proportional to the coupling constant of a glueball to two pseudoscalars at rest. If the $\kappa$ is chosen so that $E_{2}^{\pi \pi}=E^{g}$, however, the lightest intermediate state which can appear between the glueball and pseudoscalars in $T_{2}\left(t_{g}, t_{\pi}\right)$ is still $\mid 1>$, not $\mid 2>$, barring the unlikely occurrence of $\eta_{12}^{\pi \pi}(t)=0$. To get the coupling of a glueball to two pseudoscalars with momenta of magnitude $2 \pi L^{-1}$ from $T_{2}\left(t_{g}, t_{\pi}\right)$, the contribution to $T_{2}\left(t_{g}, t_{\pi}\right)$ from the $\mid 1>$ intermediate state must be removed.

We therefore define the subtracted amplitudes

$S_{i}\left(t_{g}, t_{\pi}\right)=T_{i}\left(t_{g}, t_{\pi}\right)-\frac{\eta_{j i}^{\pi \pi}\left(t_{\pi}\right)}{\eta_{j j}^{\pi \pi}\left(t_{\pi}\right)} T_{j}\left(t_{g}, t_{\pi}\right)$,

for $(i, j)$ of either $(1,2)$ or $(2,1)$. In $S_{i}\left(t_{g}, t_{\pi}\right)$ the contribution from the intermediate state $|j\rangle$ has been canceled. Although the subtraction in $S_{1}\left(t_{g}, t_{\pi}\right)$ is irrelevant for large enough $t_{g}$, we expect the subtracted $S_{1}\left(t_{g}, t_{\pi}\right)$ to approach its large $t_{g}$ behavior more rapidly than $T_{1}\left(t_{g}, t_{\pi}\right)$ approaches its large $t_{g}$ limit.

Another state which can also appear between the pseudoscalars and glueball in $T_{i}\left(t_{g}, t_{\pi}\right)$ consists of a quark and an antiquark bound as a scalar flavor singlet. For the lattice size, $\beta$ and $\kappa$ used in the present calculation, this state we have found has a mass in lattice units above 1.25. The scalar glueball mass is $0.972(44)$. The scalar quark-antiquark state therefore will make only its appropriate virtual contribution and does not require an additional correction.

For $t_{g}$ and $t_{\pi}$ large with $\left(t_{g}+t_{\pi}\right) \ll T$, the three-point functions become

$S_{i}\left(t_{g}, t_{\pi}\right) \rightarrow \frac{c_{i} \sqrt{3} \lambda_{i} \eta^{g} \eta_{i i}^{\pi \pi}(1-r) L^{3}}{\sqrt{8 E^{g}\left(E_{i}^{\pi}\right)^{2}}} s_{i}\left(t_{g}, t_{\pi}\right)$.

Here $c_{1}=1 / \sqrt{2}, c_{2}=\sqrt{3}, r$ is $\left(\eta_{12}^{\pi \pi} \eta_{21}^{\pi \pi}\right) /\left(\eta_{11}^{\pi \pi} \eta_{22}^{\pi \pi}\right)$ and $\lambda_{1}$ and $\lambda_{2}$ are the glueball coupling constants to a pair of pseudoscalars at rest or with momenta of magnitude $2 \pi L^{-1}$, respectively. As discussed earlier, $\eta_{i j}^{\pi \pi}$ is defined from $\eta_{i j}^{\pi \pi}(t)$ as large $t$. The factor $s_{i}\left(t_{g}, t_{\pi}\right)$ is

$$
\begin{aligned}
s_{i}\left(t_{g}, t_{\pi}\right)= & \sum_{t} \exp \left[-E^{g}\left|t-t_{g}\right|-E_{i}^{\pi}|t|-\right. \\
& \left.E_{i}^{\pi}\left|t-t_{\pi}\right|-\delta_{i}\left(t, t_{\pi}\right)\left|t-t_{\pi}\right|\right] .
\end{aligned}
$$

For $t \geq t_{\pi}, \delta_{i}\left(t, t_{\pi}\right)$ is the binding energy $E_{i}^{\pi \pi}-$ $2 E_{i}^{\pi}$ and otherwise $\delta_{i}\left(t, t_{\pi}\right)$ is 0 . 
The coupling constants in Eq. (2) have been identified by fitting $S_{i}\left(t_{g}, t_{\pi}\right)$ with the three-point function arising from a simple phenomenological interaction lagrangian. This procedure for determining $\lambda_{i}$ is correct to leading order in $\lambda_{i}$. A corresponding relation has been used for some time to find coupling constants among hadrons containing quarks and recently has produced a variety of results in good agreement with experiment 11. The normalization of the $\lambda_{i}$ is chosen so that in the continuum limit they become, up to a factor of $-i$, Lorentz-invariant decay amplitudes with the standard normalization convention used in the section on kinematics of the Review of Particle Properties.

To extract $\lambda_{i}$ from $S_{i}\left(t_{g}, t_{\pi}\right)$ using Eq. (2) we need the $\eta_{i j}^{\pi \pi}$. These we obtain from propagators for two-pseudoscalar states. Define $C_{i}\left(t_{1}, t_{2}\right)$ to be $<\Pi\left(t_{1}+2 t_{2}, t_{1}+t_{2}\right) \tilde{\Pi}_{i}\left(t_{2}, 0\right)>$. For moderately large values of $t_{1}$, we then have

$$
\begin{aligned}
C_{i}\left(t_{1}, t_{2}\right)= & C_{1 i}\left(t_{2}\right) \exp \left(-E_{1}^{\pi \pi} t_{1}\right)+ \\
& C_{2 i}\left(t_{2}\right) \exp \left(-E_{2}^{\pi \pi} t_{1}\right)+D_{i}\left(t_{2}\right), \\
C_{i j}\left(t_{2}\right)= & \eta_{i 1}^{\pi \pi}\left(t_{2}\right) \eta_{i j}^{\pi \pi}\left(t_{2}\right)+ \\
& \sqrt{6} \eta_{i 2}^{\pi \pi}\left(t_{2}\right) \eta_{i j}^{\pi \pi}\left(t_{2}\right) .
\end{aligned}
$$

The term $D_{i}\left(t_{2}\right)$, independent of $t_{1}$, arises from propagation across the lattice's periodic time direction boundary. $D_{i}\left(t_{2}\right)$ makes a significant contribution to $C_{i}\left(t_{1}, t_{2}\right)$ only if $t_{1}+t_{2}$ is comparable to $T$. From Eqs. (画) and (5), $\eta_{i j}^{\pi \pi}(t)$ and $\eta_{i j}^{\pi \pi}$ can be found.

One of the jobs performed by the $\eta_{i j}^{\pi \pi}$ in Eq. (2) is to correct for the interaction between the two pseudoscalars produced by a glueball decay. In the valence approximation pseudoscalars interact without the production and annihilation of virtual quark-antiquark pairs. The $C_{i}\left(t_{1}, t_{2}\right)$ from which the $\eta_{i j}^{\pi \pi}$ are obtained should therefore be evaluated from quark propagators including only terms in which all initial quarks and antiquarks propagate through to final quarks and antiquarks. It can be shown that including also in the twopseudoscalar propagator terms in which initial quarks propagate to initial antiquarks and final quarks propagate to final antiquark would lead to $\eta_{i j}^{\pi \pi}$ which correct Eq. (2) for processes missing from glueball decay in the valence approximation.

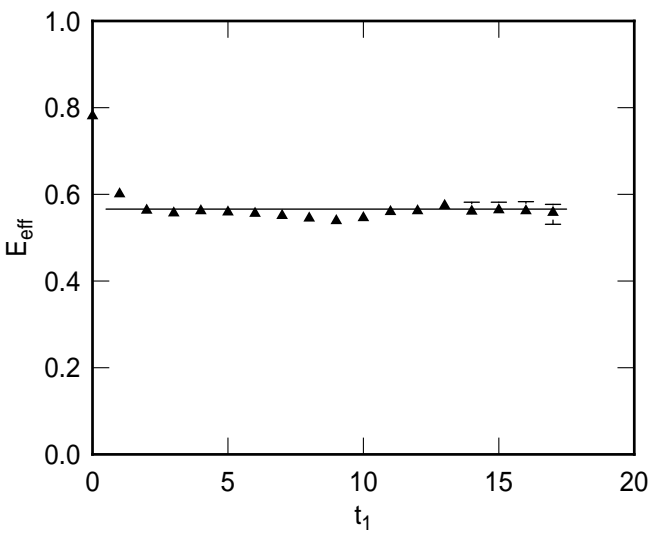

Figure 2. Effective masses determined from the $t_{1}$ dependence of $C_{1}\left(t_{1}, t_{2}\right)-D_{1}\left(t_{2}\right)$ for $\kappa=0.1675$ and $t_{2}=2$.

If $t_{1}$ is made very large, the $C_{i}\left(t_{1}, t_{2}\right)$ are given by a sum of two terms each of which is a slightly more complicated version of one of the exponentials in Eq. (4). This complication occurs because in the valence approximation the exchange of a particle composed of quarks and antiquarks between the pseudoscalars produced in a glueball decay is not iterated in the same way as in full QCD. The term in Eq. (11) with coefficient $C_{j i}\left(t_{2}\right)$ requires no modification if $\left|E_{j}^{\pi \pi}-2 E_{j}^{\pi}\right|^{2} t_{1}^{2} / 2 \ll 1$. Within the intervals of $t_{1}$ we use to determine the $\eta_{i j}^{\pi \pi}$, these bounds are well satisfied. In any case, as we will discuss below, the values we obtain for $\eta_{i j}^{\pi \pi}$ turn out to be close to their values for noninteracting pseudoscalars. A consequence is that the corrections due to interactions between the decay pseudoscalars which the $\eta_{i j}^{\pi \pi}$ contribute to the predicted values of $\lambda_{i}$ are small, and our results are fairly insensitive to details of the twopseudoscalar interaction.

\section{Results}

We now turn to our numerical results. In all of our numerical work, fits were done by minimizing $\chi^{2}$ found from the full correlation matrix among the fitted data and statistical uncertainties were found by the bootstrap method.

At $\beta=5.7$ on a $16^{3} \times 24$ lattice, with an ensemble of 10500 independent configurations, we determined glueball and single pseudoscalar energies and renormalization constants following Refs. [1] and [6], respectively. The energies in 


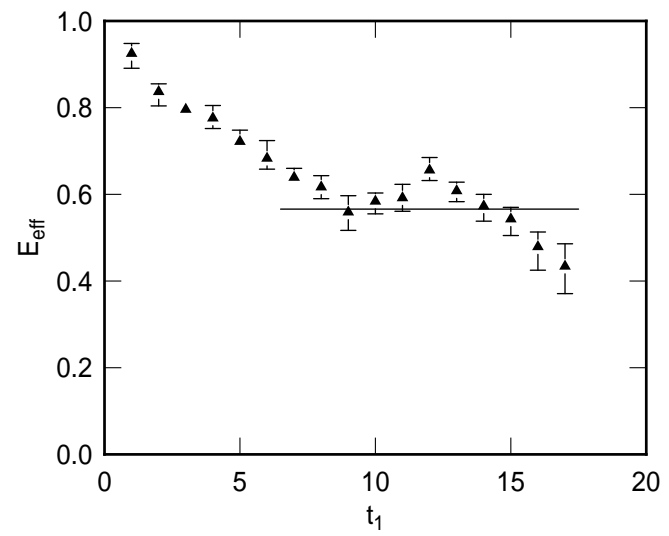

Figure 3. Effective masses determined from the $t_{1}$ dependence of $C_{2}\left(t_{1}, t_{2}\right)-D_{2}\left(t_{2}\right)$ for $\kappa=0.1675$ and $t_{2}=2$.

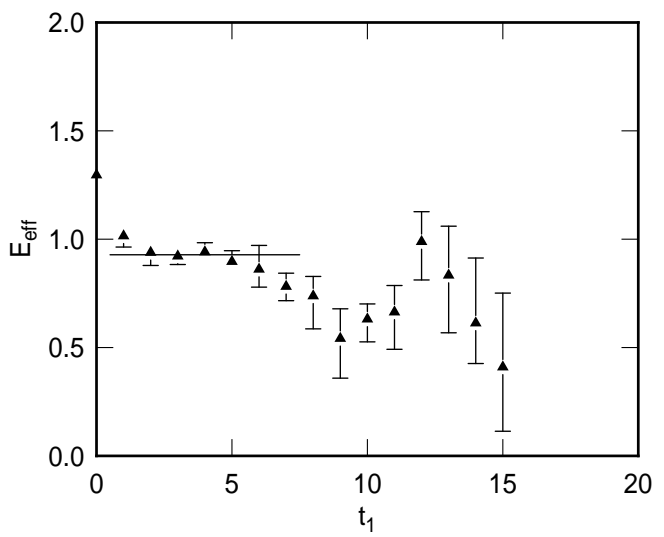

Figure 4. Effective masses determined from the $t_{1} \quad$ dependence of $C_{2}\left(t_{1}, t_{2}\right)-C_{12}\left(t_{2}\right) \exp \left(-E_{1}^{\pi \pi} t_{1}\right)-D_{2}\left(t_{2}\right)$ for $\kappa=0.1675$ and $t_{2}=2$.
Single particle energies in lattice units.

lattice units are shown in Table 1 .

On a lattice of size $16^{3} \times 40$ we then evaluated the two-pseudoscalar propagator $C_{i}\left(t_{1}, t_{2}\right)$ at $\kappa=0.1650$ using 107 independent configuration, and at $\kappa=0.1675$ using 875 independent configurations. Fitting the $t_{1}$ dependence of $C_{i}\left(t_{1}, t_{2}\right)$ to Eqs. (位) and (5), we determined $E_{i}^{\pi \pi}$ and $\eta_{i j}^{\pi \pi}\left(t_{2}\right)$ for a range of different $t_{2}$. For each value of $\kappa$ we first found $E_{1}^{\pi \pi}$ by fitting the $t_{1}$ dependence of $C_{1}\left(t_{1}, t_{2}\right)$ with fixed $t_{2}$ to Eq. (4). This fit was done at large enough $t_{1}$ for the contribution of the more rapidly falling term $C_{21}\left(t_{2}\right) \exp \left(-E_{2}^{\pi \pi} t_{1}\right)$ in Eq. (14) to be negligible. Since the coefficient $C_{21}\left(t_{2}\right)$ is significantly smaller than $C_{11}\left(t_{2}\right)$, it is not difficult to find a range of $t_{1}$ within which only the $C_{11}\left(t_{2}\right)$ term survives. For both values of $\kappa$ we found $11-t_{2} \leq t_{1} \leq 18-t_{2}$ to be satisfactory. Figure 2 shows the fit to $C_{1}\left(t_{1}, t_{2}\right)$ for $\kappa=0.1675, t_{2}=2$. The horizontal line is the fitted value of $E_{1}^{\pi \pi}$. The points with error bars are effective energies $E_{\text {eff }}$ given by $\log \left[c_{1}\left(t_{1}, t_{2}\right) / c_{1}\left(t_{1}+1, t_{2}\right)\right]$ for $c_{1}\left(t_{1}, t_{2}\right)$ defined as the propagator with constant term removed $C_{1}\left(t_{1}, t_{2}\right)-D_{1}\left(t_{2}\right)$.

We then looked for a region at large $t_{1}$ in which $C_{2}\left(t_{1}, t_{2}\right)$ gets negligible contribution from $C_{22}\left(t_{2}\right) \exp \left(-E_{2}^{\pi \pi} t_{1}\right)$ in Eq. (4). This region is more difficult to locate than the corresponding region for $C_{1}\left(t_{1}, t_{2}\right)$, since the coefficient $C_{12}\left(t_{2}\right)$ of the dominant large $t_{1}$ term in Eq. (4) is smaller than the coefficient $C_{22}\left(t_{2}\right)$ of the term which falls more rapidly at large $t_{1}$. In these fits, we therefore took as input the value already determined for $E_{1}^{\pi \pi}$ from the fit to $C_{1}\left(t_{1}, t_{2}\right)$. A satisfactory range for both $\kappa$ we found to be $14-t_{2} \leq t_{1} \leq$ $19-t_{2}$. For the fit to $C_{2}\left(t_{1}, t_{2}\right)$ with $\kappa=0.1675$, $t_{2}=2$, Figure 3 shows $E_{1}^{\pi \pi}$ in comparison to effective energies determined from $c_{2}\left(t_{1}, t_{2}\right)$ defined as $C_{2}\left(t_{1}, t_{2}\right)-D_{2}\left(t_{2}\right)$. With $C_{12}\left(t_{2}\right)$ determined, we 
Table 2

\begin{tabular}{cll}
\hline \multicolumn{1}{c}{$\kappa$} & & \\
\hline 0.1650 & $E_{1}^{\pi \pi}$ & $0.9076(49)$ \\
& $E_{2}^{\pi \pi}$ & $1.2180(155)$ \\
\hline 0.1675 & $E_{1}^{\pi \pi}$ & $0.5690(44)$ \\
& $E_{2}^{\pi \pi}$ & $0.8925(243)$ \\
\hline \multicolumn{2}{c}{$\hat{\eta}_{i j}^{\pi \pi}$} \\
\hline 0.1650 & $0.988(30)$ & $0.091(8)$ \\
& $-0.087(8)$ & $1.065(13)$ \\
\hline 0.1675 & $1.050(21)$ & $0.107(6)$ \\
& $-0.112(8)$ & $1.053(53)$ \\
\hline
\end{tabular}

Energies in lattice units and field-strength renormalizations for two-pseudoscalar states.

then formed the subtracted propagator $d_{2}\left(t_{1}, t_{2}\right)$ given by $C_{2}\left(t_{1}, t_{2}\right)-C_{12}\left(t_{2}\right) \exp \left(-E_{1}^{\pi \pi} t_{1}\right)-D_{2}\left(t_{2}\right)$ and looked for a plateau in effective masses at smaller $t_{1}$ to be fit to $C_{22}\left(t_{2}\right) \exp \left(-E_{2}^{\pi \pi} t_{1}\right)$. A satisfactory range we found to be the 4 successive time values in the interval $2 \leq t_{1} \leq 7$ giving the fit with the smallest $\chi^{2}$ per degree of freedom. For the fit to $d_{2}\left(t_{1}, t_{2}\right)$ with $\kappa=0.1675, t_{2}=2$, Figure 4 shows $E_{2}^{\pi \pi}$ determined from $3 \leq t_{1} \leq 6$ in comparison to effective energies determined from $d_{2}\left(t_{1}, t_{2}\right)$.

At $\kappa=0.1650$ we obtained results for $0 \leq t_{2} \leq$ 4 , and at 0.1675 we found results for $t_{2}$ of 0 and $2 \leq t_{2} \leq 5$. The values of $E_{i}^{\pi \pi}$ were statistically consistent with being independent of $t_{2}$ in all cases. The $\eta_{i j}^{\pi \pi}\left(t_{2}\right)$ were consistent with the asymptotic form $\eta_{i j}^{\pi \pi} \exp \left(-E_{j}^{\pi} t_{2}\right)$ in all cases for $t_{2} \geq 2$. The final values of $E_{i}^{\pi \pi}$ and $\hat{\eta}_{i j}^{\pi \pi}$ used in finding the $\lambda_{i}$ were taken from combined fits with $2 \leq t_{2} \leq 4$ for $\kappa=0.1650$ and with $2 \leq t_{2} \leq 5$ for $\kappa=0.1675$. Table 2 gives the final $E_{i}^{\pi \pi}$ in lattice units and $\hat{\eta}_{i j}^{\pi \pi}$. For noninteracting pseudoscalars $\hat{\eta}_{i j}^{\pi \pi}$ is 1 for $i=j$ and 0 otherwise. Our data is close to these values. The final value of $\lambda_{1}$ is changed by less than 1 standard deviation and the final $\lambda_{2}$ is changed by less than 2 standard deviations if we ignore the determination of $\hat{\eta}_{i j}^{\pi \pi}$ and simply use the the noninteracting values.

From our fitting procedure we were unable to obtain a satisfactory signal for the coefficient $C_{21}\left(t_{2}\right)$ which enters the determination of $\hat{\eta}_{21}^{\pi \pi}$ and therefore of $\lambda_{1}$. Since the other $\eta_{i j}^{\pi \pi}$ are close to their values for pairs of noninteracting pseudoscalars, we took $\hat{\eta}_{21}^{\pi \pi}$ from the rela-

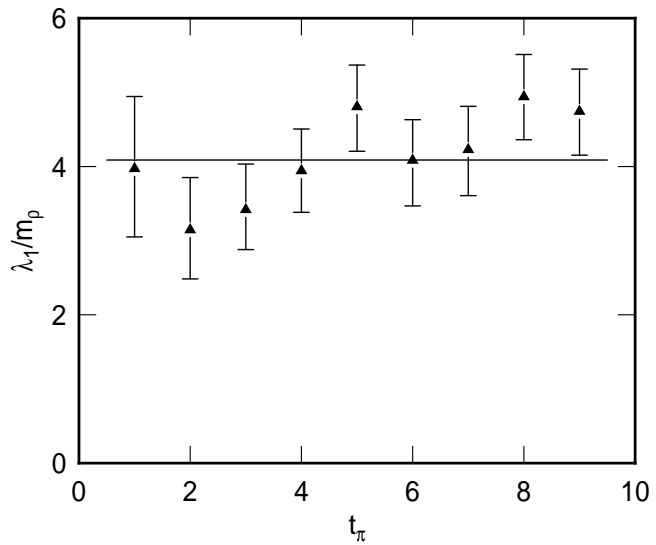

Figure 5. $\lambda_{1}$ for $t_{g}-t_{\pi}=0$ as a function of $t_{\pi}$, compared with a fit on $t_{g}-t_{\pi}=0,3 \leq t_{\pi} \leq 7$.

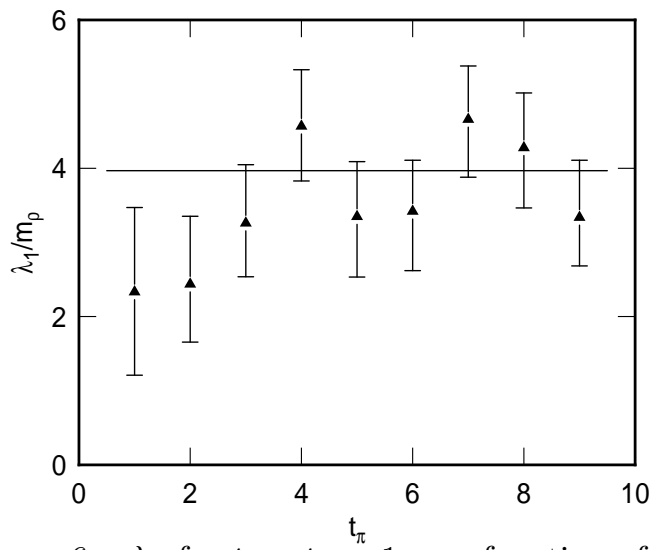

Figure 6. $\lambda_{1}$ for $t_{g}-t_{\pi} \stackrel{t_{\pi}}{=} 1$ as a function of $t_{\pi}$, compared with a fit on $t_{g}-t_{\pi}=1,3 \leq t_{\pi} \leq 7$.

tion $\hat{\eta}_{21}^{\pi \pi} / \hat{\eta}_{22}^{\pi \pi}=-\hat{\eta}_{12}^{\pi \pi} / \hat{\eta}_{11}^{\pi \pi}$ which follows from first order perturbation theory in the strength of the two-pseudoscalar interaction. This approximation should introduce only a small error since simply setting $\hat{\eta}_{21}^{\pi \pi}$ to 0 alters the final $\lambda_{1}$ by less than a standard deviation. As discussed earlier, if $\lambda_{1}$ is determined from Eq. (2) at large enough $t_{g}-t_{\pi}$, the result is completely independent of $\hat{\eta}_{21}^{\pi \pi}$.

From our 10500 configuration ensemble on a $16^{3} \times 24$ lattice, we evaluated $S_{1}$ and $S_{2}$ for glueball decay on mass shell at $\kappa$ of 0.1650 and 0.1675 , respectively. We obtained statistically significant results for $0 \leq t_{g}-t_{\pi} \leq 2$ with $0 \leq t_{\pi} \leq 9$ for $S_{1}$ and with $0 \leq t_{\pi} \leq 7$ for $S_{2}$. At each point within this range we then determined effective $\lambda_{i}$ using Eq. (2). We found $\lambda_{1}$ and $\lambda_{2}$ statistically consistent with being constant for $t_{\pi} \geq 3$ and $t_{\pi} \geq 2$, 


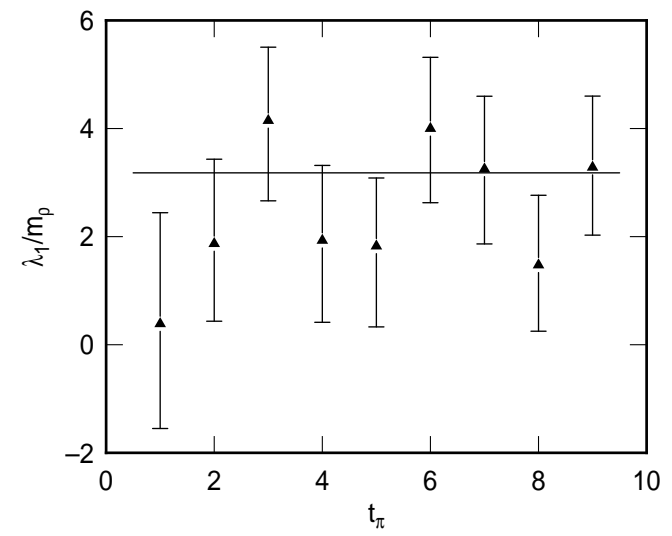

Figure 7. $\lambda_{1}$ for $t_{g}-t_{\pi}=2$ as a function of $t_{\pi}$, compared with a fit on $t_{g}-t_{\pi}=2,3 \leq t_{\pi} \leq 7$.

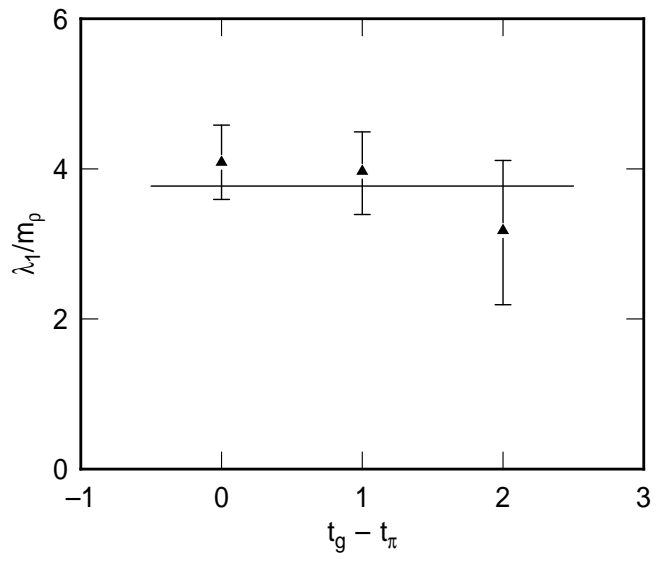

Figure 8. $\quad \lambda_{1}$ fitted on intervals $3 \leq t_{\pi} \leq 7$ with a single $t_{g}-t_{\pi}$ as a function of $t_{g}-t_{\pi}$, compared with a fit on $1 \leq t_{g}-t_{\pi} \leq 2,3 \leq t_{\pi} \leq 7$.

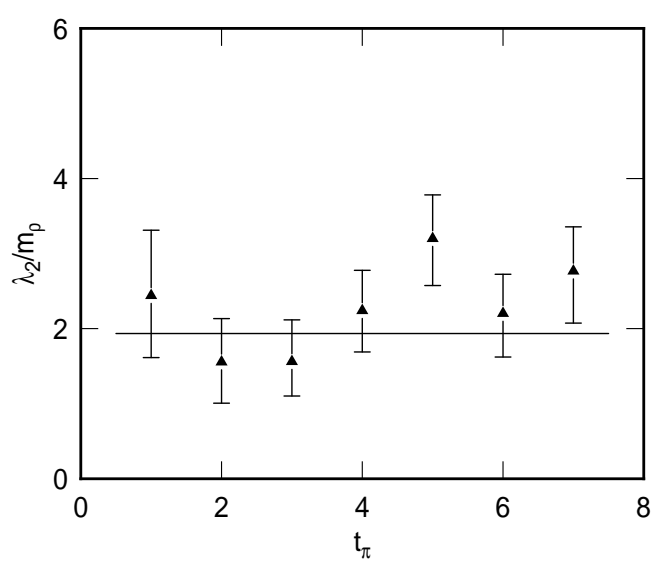

Figure 9. $\lambda_{2}$ for $t_{g}-t_{\pi}=0$ as a function of $t_{\pi}$, compared with a fit on $t_{g}-t_{\pi}=0,2 \leq t_{\pi} \leq 6$.

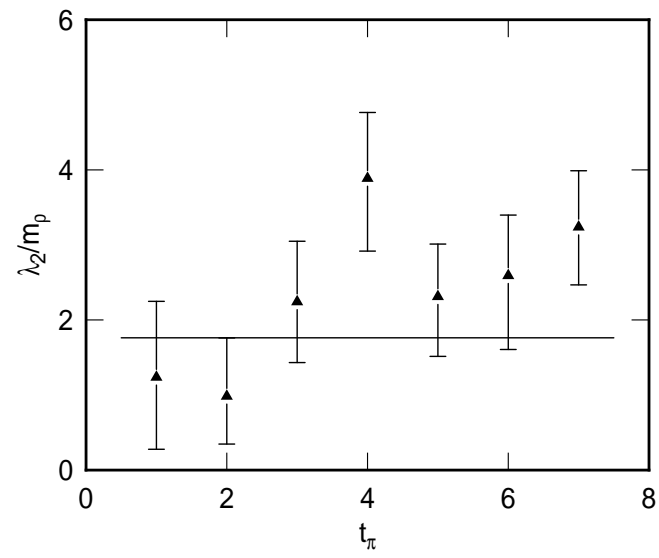

Figure 10. $\lambda_{2}$ for $t_{g}-t_{\pi}=1$ as a function of $t_{\pi}$, compared with a fit on $t_{g}-t_{\pi}=1,2 \leq t_{\pi} \leq 6$.

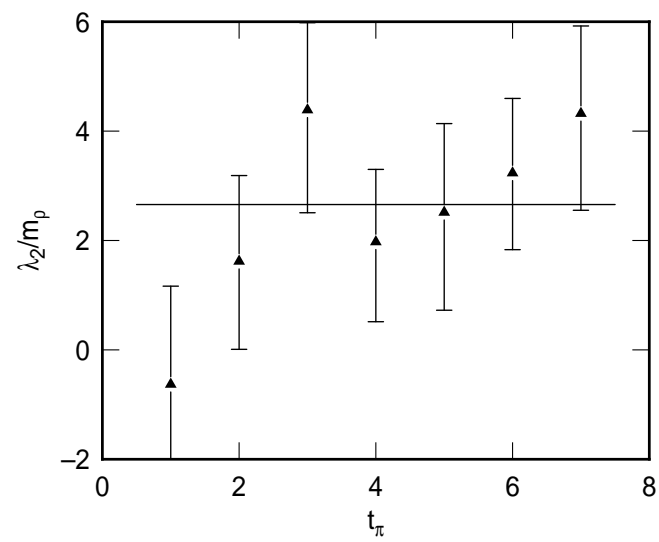

Figure 11. $\lambda_{2}$ for $t_{g}-t_{\pi}=2$ as a function of $t_{\pi}$, compared with a fit on $t_{g}-t_{\pi}=2,2 \leq t_{\pi} \leq 6$.

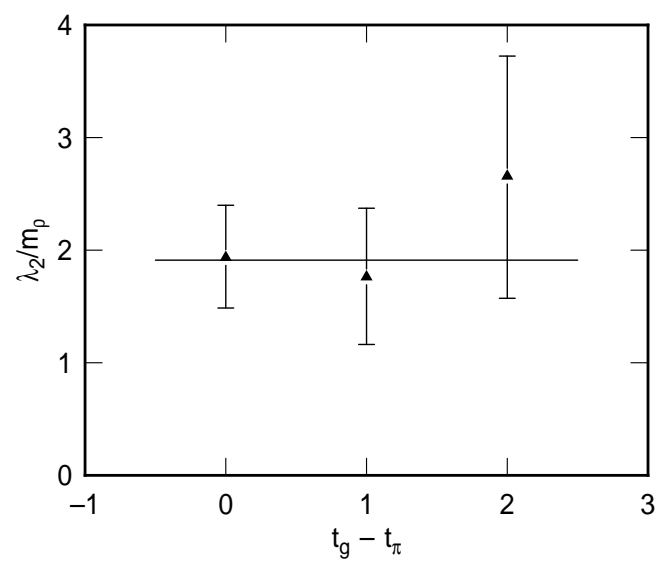

Figure 12. $\quad \lambda_{1}$ fitted on intervals $3 \leq t_{\pi} \leq 7$ with a single $t_{g}-t_{\pi}$ as a function of $t_{g}-t_{\pi}$, compared with a fit on $0 \leq t_{g}-t_{\pi} \leq 1,2 \leq t_{\pi} \leq 6$. 
respectively, and all values of $t_{g}-t_{\pi}$. Figures 5 shows effective $\lambda_{1}$ in units of the $\rho$ mass as a function of $t_{\pi}$ for $t_{g}-t_{\pi}=0$ in comparison a fit with $3 \leq t_{\pi} \leq 7, t_{g}-t_{\pi}=0$. Figures 6 and 7 show corresponding data for $t_{g}-t_{\pi}$ of 1 and 2 , respectively. Figure 8 shows fitted values of $\lambda_{1}$ on the interval $3 \leq t_{\pi} \leq 7$ for fixed $t_{g}-t_{\pi}$ of 0,1 or 2 . Figures 9 shows effective $\lambda_{2}$ in units of the $\rho$ mass as a function of $t_{\pi}$ for $t_{g}-t_{\pi}=0$ in comparison a fit with $2 \leq t_{\pi} \leq 6, t_{g}-t_{\pi}=0$. Figures 10 and 11 show corresponding data for $t_{g}-t_{\pi}$ of 1 and 2 , respectively. Figure 12 shows fitted values of $\lambda_{1}$ on the interval $2 \leq t_{\pi} \leq 6$ for fixed $t_{g}-t_{\pi}$ of 0,1 or 2 .

To extract final values of $\lambda_{i}$, we tried fits to all rectangular intervals of data including at least 4 values of $t_{\pi}$ and at least 2 values of $t_{g}-t_{\pi}$. For each $\lambda_{i}$ we chose the fit giving the lowest value of $\chi^{2}$ per degree of freedom. The window determined in this way for $\lambda_{1}$ is $3 \leq t_{\pi} \leq 7$ with $1 \leq t_{g}-t_{\pi} \leq 2$, and for $\lambda_{2}$ is $2 \leq t_{\pi} \leq 6$ with $0 \leq t_{g}-t_{\pi} \leq 1$. The horizontal lines in Figures 8 and 12 shows the final value of $\lambda_{1}$ and $\lambda_{2}$ respectively. Over the full collection of windows we examined, the fitted results varied from our final results by at most 1 standard deviation. We believe our best fits provide reasonable estimates of the asymptotic coefficients in Eq. (2).

So far our discussion has been restricted to QCD with $\mathrm{u}, \mathrm{d}$ and $\mathrm{s}$ quark masses degenerate. An expansion to first order in the quark mass matrix taken around some relatively heavy $\mathrm{SU}(3)$ symmetric point gives glueball decay couplings for $\pi$ 's, K's and the $\eta$ which are a common linear function of each meson's average quark mass. Since meson masses squared are also nearly a linear function of average quark mass, the decay couplings are a linear function of meson masses squared. Thus from a linear fit to our predictions for decay couplings as a function of pseudoscalar mass squared at unphysical degenerate values of quark masses we can extrapolate decay couplings for physical nondegenerate values of quark masses. From this linear fit a prediction can also be made for the decay coupling of the scalar glueball to $\eta+\eta^{\prime}$, if we ignore the contribution to the decay from the process in which the $\eta$ quark and antiquark are connected to each other by one propagator and the $\eta^{\prime}$ quark and antiquark are connected to each other by a second propagator.

Figure 1 shows predicted coupling constants as a function of predicted meson mass squared along with linear extrapolations of the predicted values to the physical $\pi, \mathrm{K}$ and $\eta$ masses, in comparison to observed decay couplings [8] for decays of $f_{J}(1710)$ to pairs of $\pi$ 's, K's and $\eta$ 's. Masses and decay constants are shown in units of the $\rho$ mass. Our predicted width for the scalar glueball decay to $\eta+\eta^{\prime}$ is $6(3) \mathrm{MeV}$. For the ratio $\lambda_{\eta \eta^{\prime}} / \lambda_{\eta \eta}$ we get $0.52(13)$. We predict a total width for glueball decay to pseudoscalar pairs of $108(29) \mathrm{MeV}$, in comparison to $99(15) \mathrm{MeV}$ for $f_{J}(1710)$.

One of us (D.W.) is grateful to S. Lindenbaum, R. Longacre, S. Sharpe and the participants in Gluonium 95 for valuable conversations.

\section{REFERENCES}

1. H. Chen, J. Sexton, A. Vaccarino and D. Weingarten, Nucl. Phys. B (Proc. Suppl.) 34, 357 (1994).

2. G. Bali, K. Schilling, A. Hulsebos, A. Irving, C. Michael, P. Stephenson, Phys. Lett. B 309, 378 (1993).

3. D. Weingarten, Nucl. Phys. B (Proc. Suppl.) 34, 29 (1994).

4. C. Amsler, et al., Phys. Lett. B355, 425 (1995).

5. D. Weingarten, Phys. Lett. 109B, 57 (1982).

6. F. Butler, H. Chen, J. Sexton, A. Vaccarino, and D. Weingarten, Nucl. Phys. B 430, 179 (1994); Nucl. Phys. B 421, 217 (1994).

7. C. Amsler and F. Close, Phys. Lett. B353, 385 (1995).

8. S. Lindenbaum and R. Longacre, Phys. Letts. B274, 494 (1992).

9. D. Weingarten, Nucl. Phys. B (Proc. Suppl.) 17, 272 (1990).

10. J. Sexton, A. Vaccarino and D. Weingarten, Nucl. Phys. B (Proc. Suppl.) 42, 279 (1995).

11. S. Gottlieb, et al., Phys. Lett. 134B, 346 (1984) R. Altmeyer, et al., Nucl. Phys. B (Proc. Suppl.) 34, 373 (1994); K. Liu, et al. Phys. Rev. Lett. 74, 2172 (1995) 